PROCEEDINGS OF THE

AMERICAN MATHEMATICAL SOCIETY

Volume 125, Number 7, July 1997, Pages 2073-2082

S 0002-9939(97)03817-3

\title{
ON $p$-SUMMABLE SEQUENCES IN THE RANGE OF A VECTOR MEASURE
}

\author{
CÁNDIDO PIÑEIRO
}

(Communicated by Palle E. T. Jorgensen)

\begin{abstract}
Let $p>2$. Among other results, we prove that a Banach space $X$ has the property that every sequence $\left(x_{n}\right) \in \ell_{u}^{p}(X)$ lies inside the range of an $X$-valued measure if and only if, for all sequences $\left(x_{n}^{*}\right)$ in $X^{*}$ satisfying that the operator $x \in X \rightarrow\left(\left\langle x, x_{n}^{*}\right\rangle\right) \in \ell_{1}$ is 1-summing, the operator $x \in X \rightarrow$ $\left(\left\langle x, x_{n}^{*}\right\rangle\right) \in \ell_{q}$ is nuclear, being $q$ the conjugate number for $p$. We also prove that, if $X$ is an infinite-dimensional $\mathcal{L}_{p}$-space for $1 \leq p<2$, then $X$ can't have the above property for any $s>2$.
\end{abstract}

\section{INTRODUCTION}

Let $X$ be a Banach space and let $p>2$. In [Pi2] it is proved that every sequence $\left(x_{n}\right)$ in $X$ satisfying $\sum_{n}\left|\left\langle x_{n}, x^{\star}\right\rangle\right|^{p}<+\infty$ for all $x^{\star} \in X^{\star}$ lies inside the range of an $X$-valued measure with bounded variation if and only if $X$ is finite-dimensional. On the other hand, in $[\mathrm{AD}]$ the authors proved that for every Banach space and for every sequence $\left(x_{n}\right) \in \ell_{w}^{2}(X)$ there is an $X$-valued countably additive measure whose range contains $\left(x_{n}\right)$.

The purpose of this paper is to characterize, given a real number $p>2$, the Banach spaces $X$ in which every sequence $\left(x_{n}\right) \in \ell_{u}^{p}(X)$ lies inside the range of an $X$-valued countably additive measure.

\section{NOTATION}

We start by explaining some basic notation used in this paper. In general, our operator and vector measure terminology and notation follow $[\mathrm{T}]$ and [DU]. We only consider real Banach spaces. If $X$ is a such space, $B_{X}$ will denote its closed unit ball. The phrase "range of an $X$-valued measure" always means a set of the form $\operatorname{rg}(F)=\{F(A): A \in \Sigma\}$, where $\Sigma$ is a $\sigma$-algebra of subsets of a set $\Omega$ and $F: \Sigma \rightarrow X$ is countably additive. We denote by $\|F\|$ its total semivariation

$$
\|F\|=\sup \left\{\left|x^{*} \circ F\right|(\Omega): x^{*} \in B_{X^{*}}\right\} .
$$

Given $p \geq 1, \ell_{w}^{p}(X)$ will denote the vector space of all sequences $\left(x_{n}\right)$ in $X$ such that $\sum_{n=1}^{\infty}\left|\left\langle x_{n}, x^{\star}\right\rangle\right|^{p}<+\infty$ for all $x^{\star} \in X^{\star}$. It is easy to see that if $\left(x_{n}\right) \in \ell_{w}^{p}(X)$

Received by the editors November 30, 1995 and, in revised form, January 31, 1996.

1991 Mathematics Subject Classification. Primary 46G10; Secondary 47B10.

(C)1997 American Mathematical Society 
then

$$
\epsilon_{p}\left(\left(x_{n}\right)\right)=\sup \left\{\left(\sum_{n=1}^{\infty}\left|\left\langle x_{n}, x^{\star}\right\rangle\right|^{p}\right)^{\frac{1}{p}}: x^{*} \in B_{X^{*}}\right\}<+\infty
$$

and $\left(\ell_{w}^{p}(X), \epsilon_{p}\right)$ is itself a Banach space.

If $\widehat{x}=\left(x_{n}\right) \in \ell_{w}^{p}(X)$ and $P$ is a finite subset of $\mathbb{N}, \widehat{x}(P)=\left(x_{n}(P)\right)$ is the sequence defined by

$$
x_{n}(P)= \begin{cases}x_{n}, & \text { for } n \in P, \\ 0, & \text { for } n \notin P\end{cases}
$$

for all $n \in \mathbb{N}$. $\ell_{u}^{p}(X)$ will denote the subspace of $\ell_{w}^{p}(X)$ consisting of the sequences $\widehat{x}=\left(x_{n}\right)$ such that the net $(\widehat{x}(P))_{P \in \mathcal{F}(\mathbb{N})}$ converges to $\left(x_{n}\right)$ in $\ell_{w}^{p}(X)$, where $\mathcal{F}(\mathbb{N})$ is the set of all finite subsets of $\mathbb{N}$. Recall that $\ell_{u}^{1}(X)$ is formed by the unconditionally summable sequences in $X$. We need the following Proposition that lists some privileges that membership in $\ell_{w}^{p}(X)$ entails.

Proposition A. Let $p>1$ and $X$ a Banach space. The following statements are equivalent:

(i) $\left(x_{n}\right) \in \ell_{w}^{p}(X)$.

(ii) The series $\sum_{n=1}^{\infty} \alpha_{n} x_{n}$ converges unconditionally for every sequence $\left(\alpha_{n}\right) \in$ $\ell_{q}$.

(iii) The map $\left(\alpha_{n}\right) \in \ell_{q} \rightarrow \sum_{n=1}^{\infty} \alpha_{n} x_{n} \in X$ defines a bounded operator.

Recall that an operator $T: X \rightarrow Y$ is said to be $\infty$-nuclear if there are sequences $\left(x_{n}^{*}\right)$ in $X^{*}$ and $\left(y_{n}\right)$ in $Y$ such that

$$
T x=\sum_{n=1}^{\infty}\left\langle x, x_{n}^{*}\right\rangle y_{n}(\forall x \in X), \quad \lim _{n \rightarrow \infty} x_{n}^{*}=0 \text { and } \epsilon_{1}\left(\left(y_{n}\right)\right)<+\infty .
$$

A norm is then defined by taking the infimum of all admissible products

$$
\left(\sup _{n}\left\|x_{n}^{*}\right\|\right) \epsilon_{1}\left(\left(y_{n}\right)\right) \text {. }
$$

$\mathcal{N}_{\infty}(X, Y)$ will denote the Banach space of all $\infty$-nuclear operators from $X$ into $Y$.

The SPaCes $\mathcal{R}(X)$ and $\mathcal{R}_{c}(X)$

We denote by $\mathcal{R}(X)$ the vector space of all sequences $\left(x_{n}\right)$ in $X$ so that there exists an $X$-valued measure $F$ satisfying

$$
\left\{x_{n}: n \in \mathbb{N}\right\} \subset \operatorname{rg}(F) .
$$

If $\left(x_{n}\right)$ belongs to $\mathcal{R}(X)$, we put $\left\|\left(x_{n}\right)\right\|=\inf \|F\|$, where the infimum is taken over all vector measures $F$ admissible in (1). Obviously, we have

$$
\left\|\left(x_{n}\right)\right\|_{\infty} \leq\left\|\left(x_{n}\right)\right\| \text { for all }\left(x_{n}\right) \in \mathcal{R}(X) .
$$

Using a direct sum of vector measures [KK, p.35], it is easy to prove that any absolutely summable series in $\mathcal{R}(X)$ is convergent. So, $(\mathcal{R}(X),\|\cdot\|)$ is a Banach space.

Next we are going to consider sequences in $X$ that lie inside the range of a vector measure with relatively compact range. We denote by $\mathcal{R}_{c}(X)$ the vector space of all such sequences $\left(x_{n}\right)$ in $X$. By [PR, Proposition 1.4], if $\left(x_{n}\right)$ belongs to $\mathcal{R}_{c}(X)$, there 
exists an unconditionally convergent series $\sum_{m} y_{m}$ satisfying $x_{n} \in \sum_{m}\left[-y_{m}, y_{m}\right]$ for all $n$. The set $\sum_{m}\left[-y_{m}, y_{m}\right]$ is the range of a vector measure $F$ for which

$$
\|F\| \leq 2 \sup \left\{\sum_{m=1}^{\infty}\left|\left\langle y_{m}, x^{*}\right\rangle\right|: x^{*} \in B_{X^{*}}\right\} .
$$

If $\left(x_{n}\right)$ belongs to $\mathcal{R}_{c}(X)$, we set

$$
\left\|\left(x_{n}\right)\right\|_{c}=\inf \epsilon_{1}\left(\left(y_{m}\right)\right),
$$

the infimum being taken over all unconditionally convergent series $\sum_{m} y_{m}$ such that $\left(x_{n}\right)$ is contained in $\sum_{m}\left[-y_{m}, y_{m}\right]$. Obviously, we have

$$
\left\|\left(x_{n}\right)\right\|_{\infty} \leq\left\|\left(x_{n}\right)\right\| \leq 2\left\|\left(x_{n}\right)\right\|_{c} \text { for all }\left(x_{n}\right) \in \mathcal{R}_{c}(X) .
$$

The next Proposition proves that $\mathcal{R}_{c}(X)$ can be isometrically identified to $\mathcal{N}_{\infty}\left(\ell_{1}, X\right)$.

Proposition 1. The Banach spaces $R_{c}(X)$ and $\mathcal{N}_{\infty}\left(\ell_{1}, X\right)$ are isometric.

Proof. If $\left(x_{n}\right) \in R_{c}(X)$, we define an operator $T: \ell_{1} \rightarrow X$ by $T\left(\alpha_{n}\right)=\sum_{n} \alpha_{n} x_{n}$ for all $\left(\alpha_{n}\right) \in \ell_{1}$. Given $\epsilon>0$, choose an unconditionally summable sequence $\left(y_{n}\right)$ in $X$ so that

$$
x_{n}=\sum_{m=1}^{\infty} \alpha_{m}^{n} y_{n} \text { and } \epsilon_{1}\left(\left(y_{m}\right)\right)<\epsilon+\left\|\left(x_{n}\right)\right\|_{c},
$$

where $\left|\alpha_{m}^{n}\right| \leq 1$ for all $n, m \in \mathbb{N}$. Obviously, $T \alpha=\sum_{m}\left\langle\alpha,\left(\alpha_{m}^{n}\right)_{n}\right\rangle y_{m}$.

Claim. There exist $\left(\lambda_{m}\right) \in B_{c_{o}}$ and $\left(z_{m}\right) \in \ell_{w}^{1}(X)$, such that $y_{m}=\lambda_{m} z_{m}$ for all $m \in \mathbb{N}$ and $\epsilon_{1}\left(\left(z_{m}\right)\right) \leq \epsilon+\epsilon_{1}\left(\left(y_{m}\right)\right)$.

To prove the claim, choose $p_{o} \in \mathbb{N}$ so that

$$
\sum_{p \geq p_{o}} \frac{1}{p^{2}}<\epsilon
$$

Since the sequence $\left(y_{m}\right)$ is unconditionally summable, we have

$$
\lim _{m \rightarrow \infty} \sup \left\{\sum_{k=m}^{\infty}\left|\left\langle y_{m}, x^{*}\right\rangle\right|:\left\|x^{*}\right\| \leq 1\right\}=0 .
$$

So we can determine a strictly increasing sequence of integers $\left(n_{p}\right)_{p}$, such that

$$
\sup \left\{\sum_{m \geq n_{p}}\left|\left\langle y_{m}, x^{*}\right\rangle\right|:\left\|x^{*}\right\| \leq 1\right\}<\frac{1}{p^{3}}
$$

for all $p \in \mathbb{N}$. Let $\left(\lambda_{m}\right)$ the sequence defined by

$$
\lambda_{m}= \begin{cases}1, & \text { for } m \leq n_{p_{o}} \\ \frac{1}{p}, & \text { for } n_{p}<m \leq n_{p+1} .\end{cases}
$$

and put $z_{m}=\left(\lambda_{m}\right)^{-1} y_{m}$ for all $m \in \mathbb{N}$. It is obvious that $\left(\lambda_{m}\right) \in B_{c_{o}}$. Hence, if we prove the inequality $\epsilon_{1}\left(\left(z_{m}\right)\right) \leq \epsilon+\epsilon_{1}\left(\left(y_{m}\right)\right)$, the claim will be established. Given 
$x^{*} \in B_{X^{*}}$, we have

$$
\begin{aligned}
\sum_{m=1}^{\infty}\left|\left\langle z_{m}, x^{*}\right\rangle\right| & =\sum_{m=1}^{n_{p_{o}}}\left|\left\langle y_{m}, x^{*}\right\rangle\right|+\sum_{p=p_{o}}^{\infty} \sum_{k=n_{p}+1}^{n_{p+1}}\left|\left\langle z_{k}, x^{*}\right\rangle\right| \\
& \leq \epsilon_{1}\left(\left(y_{m}\right)\right)+\sum_{p=p_{o}}^{\infty} p \sum_{k=n_{p}+1}^{n_{p+1}}\left|\left\langle y_{k}, x^{*}\right\rangle\right| \\
& \leq \epsilon_{1}\left(\left(y_{m}\right)\right)+\sum_{p \geq p_{0}} \frac{1}{p^{2}} \\
& <\epsilon+\epsilon_{1}\left(\left(y_{m}\right)\right) .
\end{aligned}
$$

Now, using the claim we can write $T$ in the form $T \alpha=\sum_{m}\left\langle\alpha, \lambda_{m}\left(\alpha_{m}^{n}\right)_{n}\right\rangle z_{m}$. Then $T \in \mathcal{N}_{\infty}\left(\ell_{1}, X\right)$ and

$\nu_{\infty}(T) \leq\left(\sup _{m}\left\|\left(\lambda_{m}\left(\alpha_{m}^{n}\right)_{n} \|_{\infty}\right) \epsilon_{1}\left(\left(z_{m}\right)\right)=\epsilon_{1}\left(\left(z_{m}\right)\right)<\epsilon_{1}\left(\left(y_{m}\right)\right)+\epsilon<2 \epsilon+\right\|\left(x_{n}\right) \|_{c}\right.$,

for all $\epsilon>0$. Therefore, we have prove that $\nu_{\infty}(T) \leq\left\|\left(x_{n}\right)\right\|_{c}$ for all sequences $\left(x_{n}\right)$ belonging to $R_{c}(X)$.

Conversely, let $\left(x_{n}\right)$ a sequence in $X$ such that the operator

$$
T:\left(\alpha_{n}\right) \in \ell_{1} \rightarrow \sum_{n=1}^{\infty} \alpha_{n} x_{n} \in X
$$

is $\infty$-nuclear. Given $\epsilon>0$, choose a null sequence $\left(\beta_{m}\right)$ in $\ell_{\infty}$ and $\left(y_{m}\right) \in \ell_{w}^{1}(X)$ so that

$$
T \alpha=\sum_{m=1}^{\infty}\left\langle\alpha, \beta_{m}\right\rangle y_{m} \text { for all } \alpha \in \ell_{1}
$$

and

$$
\left(\sup _{m}\left\|\beta_{m}\right\|\right) \epsilon_{1}\left(\left(y_{m}\right)\right)<\nu_{\infty}(T)+\epsilon .
$$

Since the sequence $\left(\left\|\beta_{m}\right\|_{\infty} y_{m}\right)$ is unconditionally summable, it follows easily that $\left(x_{n}\right)$ belongs to $R_{c}(X)$ and

$$
\left\|\left(x_{n}\right)\right\|_{c}<\epsilon+\nu_{\infty}(T)
$$

for all $\epsilon>0$. This concludes the proof.

It is well-known that with the trace duality $\mathcal{N}_{\infty}(X, Y)^{*}$ and $\Pi_{1}\left(Y, X^{* *}\right)$ can be isometrically identified, if $X^{*}$ has the approximation property. In fact, for any $S \in \Pi_{1}\left(Y, X^{* *}\right)$ the map $\phi_{S}: \mathcal{N}_{\infty}(X, Y) \rightarrow \mathbb{R}$ defined by $\phi_{S}(T)=\operatorname{tr}(S \circ T)$ is a continuous linear form on $\mathcal{N}_{\infty}(X, Y)$ and every linear form on $\mathcal{N}_{\infty}(X, Y)$ can be obtained in this way. Accordingly, $\mathcal{R}_{c}(X)^{*}$ and $\Pi_{1}\left(X, \ell_{1}^{* *}\right)$ are isometric. The next Proposition shows that every operator $T \in \Pi_{1}\left(X, \ell_{1}\right)$ also defines a continuous linear form on $\mathcal{R}(X)$.

Proposition 2. Let $X$ be a Banach space. If $\left(x_{n}^{*}\right)$ is a sequence in $X^{*}$ so that the operator $S: x \in X \rightarrow\left(\left\langle x, x_{n}^{*}\right\rangle\right) \in \ell_{1}$ is 1-summing, then the linear map $\psi_{S}: \mathcal{R}(X) \rightarrow \mathbb{R}$ defined by $\psi_{S}\left(\left(x_{n}\right)\right)=\sum_{n}\left\langle x_{n}, x_{n}^{*}\right\rangle$ for all $\left(x_{n}\right) \in \mathcal{R}(X)$ is welldefined, continuous and $\left\|\psi_{S}\right\| \leq \pi_{1}(S)$. 
Proof. Let $\left(x_{n}\right) \in \mathcal{R}(X)$. Given $\epsilon>0$, choose a vector measure $F: \Sigma \rightarrow X$ so that

$$
\left\{x_{n}: n \in \mathbb{N}\right\} \subset \operatorname{rg}(F) \text { and }\|F\|<\epsilon+\left\|\left(x_{n}\right)\right\| .
$$

Let $\mu$ a control measure for $F$ and consider the integration operator

$$
I: f \in L^{\infty}(\mu) \rightarrow \int f d F \in X
$$

The operator $S \circ I$ is 1 -summing and, hence, integral. Since $\ell_{1}$ has the RadonNikodym property, it follows that $S \circ I$ is nuclear and $\pi_{1}(S \circ I)=\nu_{1}(S \circ I)$ (see [DU, p. 174]). So is $(S \circ I)^{*}: \ell_{\infty} \rightarrow L^{\infty}(\mu)$. Then

$$
\sum_{n=1}^{\infty}\left\|(S \circ I)^{*}\left(e_{n}\right)\right\|<+\infty
$$

where $\left(e_{n}\right)$ is the unit basis of $c_{o}$. On the other hand, we have

$$
\left\langle(S \circ I) f, e_{n}\right\rangle=\left\langle f,(S \circ I)^{*}\left(e_{n}\right)\right\rangle .
$$

Then

$$
\begin{gathered}
\left\|(S \circ I)^{*}\left(e_{n}\right)\right\|=\sup \left\{\left|\left\langle f,(S \circ I)^{*}\left(e_{n}\right)\right\rangle\right|:\|f\|_{\infty} \leq 1\right\} \\
=\sup \left\{\left|\int f d\left(x_{n}^{*} \circ F\right)\right|:\|f\|_{\infty} \leq 1\right\} .
\end{gathered}
$$

This shows that (4) can be written in the form

$$
\sum_{n=1}^{\infty} \sup \left\{\left|\int f d\left(x_{n}^{*} \circ F\right)\right|:\|f\|_{\infty} \leq 1\right\}<+\infty .
$$

For every $n \in \mathbb{N}$, we choose $A_{n} \in \Sigma$ such that $F\left(A_{n}\right)=x_{n}$. Then, it follows from (5) that

$$
\sum_{n=1}^{\infty}\left|\left\langle x_{n}, x_{n}^{*}\right\rangle\right|=\sum_{n=1}^{\infty}\left|\int \chi_{A_{n}} d\left(x_{n}^{*} \circ F\right)\right|<+\infty .
$$

So, the linear form $\psi_{S}$ is well-defined. To conclude the proof we need to show that

$$
\sup \left\{\left|\sum_{n=1}^{\infty}\left\langle x_{n}, x_{n}^{*}\right\rangle\right|:\left\|\left(x_{n}\right)\right\| \leq 1\right\} \leq \pi_{1}(S) .
$$

If $B$ denotes the restriction map of $(S \circ I)^{*}$ to $c_{o}$, we have

$$
\begin{aligned}
\left|\sum_{n=1}^{\infty}\left\langle x_{n}, x_{n}^{*}\right\rangle\right| & \leq \sum_{n=1}^{\infty}\left\|(S \circ I)^{*}\left(e_{n}\right)\right\|=\nu_{1}(B) \leq \nu_{1}\left((S \circ I)^{*}\right) \leq \nu_{1}(S \circ I) \\
& =\pi_{1}(S \circ I) .
\end{aligned}
$$

Since $S \circ F$ is the representing measure of an 1-summing operator, $S \circ I$, it follows from [DU, p. 162] that $\pi_{1}(S \circ I)=|S \circ F|$ (here, $|S \circ F|$ denotes the total variation of $S \circ F)$. Finally, we can deduce from (7) that

$$
\left|\sum_{n=1}^{\infty}\left\langle x_{n}, x_{n}^{*}\right\rangle\right| \leq|S \circ F| \leq \pi_{1}(S)\|F\|<\pi_{1}(S)\left(\epsilon+\left\|\left(x_{n}\right)\right\|\right),
$$

for all $\epsilon>0$. Therefore, $\left\|\psi_{S}\right\| \leq \pi_{1}(S)$. 


\section{$p$-Summable Sequences in the RANGe of A Vector MeAsure}

Proposition 3. Let $X$ be a Banach space and let $p>2$. X has the property that every sequence $\left(x_{n}\right) \in \ell_{u}^{p}(X)$ lies inside the range of a vector measure if and only if there exists a constant $c>0$ so that, for all finite sets $\left\{x_{1}, . ., x_{n}\right\} \subset X$ satisfying $\epsilon_{p}\left(\left(x_{i}\right)_{1}^{n}\right) \leq 1$, there is a vector measure $F: \Sigma \rightarrow X$ satisfying

$$
\|F\| \leq c \text { and }\left\{x_{1}, . ., x_{n}\right\} \subset \operatorname{rg}(F) .
$$

Proof. First, proceeding by contradiction, suppose $\ell_{u}^{p}(X) \subset \mathcal{R}(X)$ but there isn't a such constant. Then there would exist a sequence $\left(H_{n}\right)$ of finite subsets of $X$ such that for every $n$ the conditions

$$
H_{n} \subset \operatorname{rg}(F) \text { and } \sup \left\{\left(\sum_{x \in H_{n}}\left|\left\langle x, x^{*}\right\rangle\right|^{p}\right)^{\frac{1}{p}}:\left\|x^{*}\right\| \leq 1\right\} \leq 1
$$

would imply $\|F\| \geq n^{2}$. If $H_{n}=\left\{x_{1}^{n}, . ., x_{k(n)}^{n}\right\}$, the sequence

$$
\left\{x_{1}^{1}, . ., x_{k(1)}^{1}, \frac{1}{2} x_{1}^{2}, . ., \frac{1}{2} x_{k(2)}^{2}, . ., \frac{1}{n} x_{1}^{n}, . ., \frac{1}{n} x_{k(n)}^{n}, . .\right\}
$$

belongs to $\ell_{u}^{p}(X)$. So, there exists a vector measure $F$ with $(1 / n) H_{n} \subset \operatorname{rg}(F)$ for all $n \in \mathbb{N}$. Thus $H_{n} \subset \operatorname{rg}(n F)$ and this would yield $\|F\| \geq n$ for every $n$, a contradiction with the fact that $F$ has bounded semivariation since it is countably additive.

Conversely, let $c>0$ be a constant such that, for every finite set $\left\{x_{1}, . ., x_{n}\right\} \subset X$ satisfying $\epsilon_{p}\left(\left(x_{i}\right)_{i=1}^{n}\right) \leq 1$, there exists a vector measure $F$ with $\|F\| \leq c$ and whose range contains $\left\{x_{1}, . ., x_{n}\right\}$. Then, the natural map from $\left(X^{(\mathbb{N})}, \epsilon_{p}\right)$ to $(\mathcal{R}(X),\|\cdot\|)$ is linear and continuous. Therefore, it has a unique continuous linear extension to $\ell_{u}^{p}(X)$.

Now we are ready to face our main result.

Theorem 4. Let $X$ be a Banach space and let $p>2$. The following are equivalent:

(i) Every sequence $\left(x_{n}\right) \in \ell_{u}^{p}(X)$ lies inside the range of a vector measure.

(ii) For all sequence $\left(x_{n}^{*}\right)$ in $X^{*}$ satisfying that $x \in X \rightarrow\left(\left\langle x, x_{n}^{*}\right\rangle\right) \in \ell_{1}$ is 1summing, the operator $x \in X \rightarrow\left(\left\langle x, x_{n}^{*}\right\rangle\right) \in \ell_{q}$ is nuclear.

(iii) There exists a constant $c>0$ such that, for all $n \in \mathbb{N}$, for all $\left\{x_{1}, . ., x_{n}\right\}$ in $X$ and all $\left\{x_{1}^{*}, . ., x_{n}^{*}\right\}$ in $X^{*}$, we have

$$
\sum_{i=1}^{n}\left|\left\langle x_{i}, x_{i}^{*}\right\rangle\right| \leq c \pi_{1}\left(\sum_{i}^{n} x_{i}^{*} \otimes e_{i}: X \rightarrow \ell_{1}^{n}\right) \epsilon_{p}\left(\left(x_{i}\right)_{i=1}^{n}\right) .
$$

Proof. (i) $\Rightarrow$ (ii) We have seen in the proof of Proposition 3 that the natural map $\ell_{u}^{p}(X) \rightarrow \mathcal{R}(X)$ is continuous. So, given a sequence $\left(x_{n}^{*}\right)$ in $X^{*}$ such that the map

$$
x \in X \rightarrow\left(\left\langle x, x_{n}^{*}\right\rangle\right) \in \ell_{1}
$$

is 1-summing, it follows from Proposition 2 that the linear form $\phi$ on $\ell_{u}^{p}(X)$ defined by $\phi\left(x_{n}\right)=\sum_{n}\left\langle x_{n}, x_{n}^{*}\right\rangle$ is continuous. Then the linear map $x \in X \rightarrow\left(\left\langle x, x_{n}^{*}\right\rangle\right) \in \ell_{q}$ is integral [DU, p. 232]. Now recall that nuclear and integral operators into a reflexive space are the same [DU].

(ii) $\Rightarrow$ (iii) The linear map

$$
T \in \Pi_{1}\left(X, \ell_{1}\right) \rightarrow I_{1 q} \circ T \in \mathcal{N}\left(X, \ell_{q}\right)
$$


has closed graph (here $I_{1 q}$ is the inclusion map from $\ell_{1}$ into $\ell_{q}$ ). So, it is continuous. Then there is a constant $c>0$ such that

$$
\nu_{1}\left(I_{1 q}^{n} \circ T\right) \leq c \pi_{1}(T)
$$

for all $n \in \mathbb{N}$ and all $T \in \mathcal{L}\left(X, \ell_{1}^{n}\right)$, being $I_{1 q}^{n}: \ell_{1}^{n} \rightarrow \ell_{q}^{n}$ the identity map. Now, given $\left\{x_{1}, . ., x_{n}\right\}$ in $X$ and $\left\{x_{1}^{*}, . ., x_{n}^{*}\right\}$ in $X^{*}$, we consider the operators $u: X \rightarrow \ell_{q}^{n}$ and $v: \ell_{q}^{n} \rightarrow X$ defined by

$$
u(x)=\left(\left\langle x, x_{i}^{*}\right\rangle\right)_{i=1}^{n} \text { and } v\left(\left(\alpha_{i}\right)\right)=\sum_{i=1}^{n} \alpha_{i} x_{i} .
$$

Since $\operatorname{tr}(u \circ v)=\sum_{1}^{n}\left\langle x_{i}, x_{i}^{*}\right\rangle$, we have

$$
\left|\sum_{i=1}^{n}\left\langle x_{i}, x_{i}^{*}\right\rangle\right| \leq \nu_{1}^{o}(u \circ v)=\nu_{1}(u \circ v) \leq \nu_{1}(u)\|v\|,
$$

being $\nu_{1}^{o}$ the finite-nuclear norm $[\mathrm{P}]$. Then, by (8) we obtain

$$
\left|\sum_{i=1}^{n}\left\langle x_{i}, x_{i}^{*}\right\rangle\right| \leq \pi_{1}\left(\sum_{i}^{n} x_{i}^{*} \otimes e_{i}: X \rightarrow \ell_{1}^{n}\right)\|v\| .
$$

This yields (iii) because of the equality $\|v\|=\epsilon_{p}\left(\left(x_{i}\right)_{i=1}^{n}\right)$.

(iii) $\Rightarrow$ (i) For every $n \in \mathbb{N}$, we define a linear map

$$
U_{n}:\left(X^{n}, \epsilon_{p}\right) \rightarrow \mathcal{N}_{\infty}\left(\ell_{1}^{n}, X\right)
$$

in the following way: Given $\widehat{x}=\left(x_{i}\right)_{1}^{n} \in X^{n}$, we denote by $T_{\widehat{x}}$ the operator from $\ell_{1}^{n}$ into $X$ defined by $T_{\widehat{x}}\left(\left(\alpha_{i}\right)_{1}^{n}\right)=\sum_{1}^{n} \alpha_{i} x_{i}$. Then we put $U_{n}(\widehat{x})=T_{\widehat{x}}$. To show that $U_{n}$ is continuous, we are going to compute $\nu_{\infty}\left(T_{\widehat{x}}\right)$ :

$$
\begin{array}{r}
\nu_{\infty}\left(T_{\widehat{x}}\right)=\sup \left\{\left|\left\langle T_{\widehat{x}}, S\right\rangle\right|: \pi_{1}(S) \leq 1, S \in \Pi_{1}\left(X, \ell_{1}^{n}\right)\right\} \\
=\sup \left\{\left|\operatorname{tr}\left(S \circ T_{\widehat{x}}\right)\right|: \pi_{1}(S) \leq 1, S \in \Pi_{1}\left(X, \ell_{1}^{n}\right)\right\} \\
=\sup \left\{\left|\sum_{i=1}^{n}\left\langle x_{i}, S^{*}\left(e_{i}^{*}\right)\right\rangle\right|: \pi_{1}(S) \leq 1, S \in \Pi_{1}\left(X, \ell_{1}^{n}\right)\right\},
\end{array}
$$

where $\left(e_{i}^{*}\right)_{1}^{n}$ is the unit basis of $\ell_{\infty}^{n}$. By (iii), we obtain

$$
\nu_{\infty}\left(T_{\widehat{x}}\right) \leq c \epsilon_{p}\left(\left(x_{i}\right)_{1}^{n}\right)
$$

So, we have

$$
\left\|U_{n}\right\| \leq c \text { for every } n \in \mathbb{N} .
$$

Now we consider the linear map

$$
U:\left(X^{(\mathbb{N})}, \epsilon_{p}\right) \rightarrow \mathcal{N}_{\infty}\left(\ell_{1}, X\right)
$$

defined by $U(\widehat{x})=T_{\widehat{x}}$ with $T_{\widehat{x}}\left(\left(\alpha_{n}\right)\right)=\sum_{n} \alpha_{n} x_{n}$, for all $\widehat{x}=\left(x_{n}\right) \in X^{(\mathbb{N})}$ and all $\left(\alpha_{n}\right) \in \ell_{1}$. From (9) it follows that $\|U\| \leq c$. Finally, Proposition 1 implies that $\ell_{u}^{p}(X)$ is contained in $\mathcal{R}_{c}(X)$.

Using the methods introduced in [Pi2], we can prove that an operator $T: X \rightarrow Y$ takes every sequence $\left(x_{n}\right) \in \ell_{u}^{p}(X)$ into a sequence $\left(T x_{n}\right)$ lying in the range of an $X$-valued measure with bounded variation if and only if $T^{*}: Y^{*} \rightarrow X^{*}$ is $(1,1, p)$ summing. The next Proposition shows the relationship between these ideas and our problem. 
Proposition 5. $\ell_{u}^{p}(X)$ is contained in $\mathcal{R}(X)$ if and only if $T^{*}: Y^{*} \rightarrow X^{*}$ is $(1,1, p)$-summing for all Banach space $Y$ and for all $T \in \Pi_{1}(X, Y)$.

Proof. Suppose $X$ is a Banach space such that $\ell_{u}^{p}(X) \subset \mathcal{R}(X)$. If $T \in \Pi_{1}(X, Y)$, then $T$ takes every sequence $\left(x_{n}\right) \in \ell_{u}^{p}(X)$ into a sequence $\left(T x_{n}\right)$ lying in the range of a vector measure of bounded variation. So, $T^{*}: Y^{*} \rightarrow X^{*}$ is $(1,1, p)$-summing.

Conversely, let $X$ be a Banach space with the property that, for all $T \in \Pi_{1}\left(X, \ell_{1}\right)$, the operator $T^{*}$ is $(1,1, p)$-summing. Take a sequence $\left(x_{n}^{*}\right)$ in $X^{*}$ such that $T: x \in$ $X \rightarrow\left(\left\langle x, x_{n}^{*}\right\rangle\right) \in \ell_{1}$ is 1 -summing. By hypothesis, $T^{*}$ is $(1,1, p)$-summing. Now an appeal to [Pi2, Theorem 1] yields the nuclearity of the operator

$$
x^{* *} \in X^{* *} \rightarrow\left(\left\langle x^{* *}, T^{*} e_{n}^{*}\right\rangle\right) \in \ell_{q} .
$$

Then $x \in X \rightarrow\left(\left\langle x, x_{n}^{*}\right\rangle\right) \in \ell_{q}$ is also nuclear. Now Theorem 4 tells us that $\ell_{u}^{p}(X) \subset \mathcal{R}(X)$.

\section{EXAMPLES}

If $X$ is a Banach space, $s(X)$ will denote the set of all real numbers $s>2$ such that every sequence $\left(x_{n}\right) \in \ell_{u}^{s}(X)$ lies inside the range of an $X$-valued measure. Obviously, $s(X)$ is an interval whose bounds are 2 and $\sup (s(X))$. In this section we find out the form of the set $s(X)$ for any $\mathcal{L}_{p}$-space $X(1 \leq p \leq+\infty)$.

a) If $X$ is an $\mathcal{L}_{p}$-space for $p \geq 2$, then $s(X)=(2,+\infty)$.

By [LR, Theorem III], $X^{*}$ is an $\mathcal{L}_{q}$-space. So $X^{*}$ is isomorphic to a subspace of an $L_{1}(\mu)$-space for some measure $\mu$ [LP, Corollary 7.2]. Hence, every null sequence in $X$ lies inside the range of an $X$-valued measure [PR].

Following [Ps2] we will say that a Banach space $X$ satisfies Grothendieck's Theorem (in short, $X$ is a $\mathrm{G}$. T. space) if $\mathcal{L}\left(X, \ell_{2}\right)=\Pi_{1}\left(X, \ell_{2}\right)$. For some time the $\mathcal{L}_{1}$-spaces remained the only known $\mathrm{G}$. T. spaces. We have just seen that $s(X)=(2,+\infty)$ for every Banach space $X$ whose dual $X^{*}$ is an $\mathcal{L}_{1}$-space. Nevertheless, there are spaces for which $s(X)=\emptyset$ and $X^{*}$ is a G. T. space. Next we will prove this assertion.

b) If $X$ is an infinite-dimensional Banach space of cotype 2 and $X^{*}$ is a G.T. space, then $s(X)=\emptyset$.

Since $X$ is a space of cotype 2 we have the identity $\Pi_{1}\left(X, \ell_{1}\right)=\Pi_{2}\left(X, \ell_{1}\right)[\mathrm{Ps} 2$, Theorem 5.16]. By [Ps2, Proposition 6.2] $\Pi_{2}\left(X, \ell_{1}\right)=\mathcal{L}\left(X, \ell_{1}\right)$. So, it follows that

$$
\pi_{1}(u) \leq c\|u\| \quad \text { for all } u \in \mathcal{L}\left(X, \ell_{1}\right)
$$

and for some constant $c>0$. Proceeding by contradiction, suppose $p \in s(X)$. Then there exists a constant $c^{\prime}>0$ so that

$$
\sum_{i=1}^{n}\left|\left\langle x_{i}, x_{i}^{*}\right\rangle\right| \leq c^{\prime} \pi_{1}\left(\sum_{i}^{n} x_{i}^{*} \otimes e_{i}: X \rightarrow \ell_{1}^{n}\right) \epsilon_{p}\left(\left(x_{i}\right)_{i=1}^{n}\right)
$$

for all $\left\{x_{i}\right\}_{i=1}^{n}$ in $X$ and all $\left\{x_{i}^{*}\right\}_{i=1}^{n}$ in $X^{*}$. (10) and (11) yield

$$
\sum_{i=1}^{n}\left|\left\langle x_{i}, x_{i}^{*}\right\rangle\right| \leq c^{\prime} c \epsilon_{p}\left(\left(x_{i}\right)_{i=1}^{n}\right) \epsilon_{1}\left(\left(x_{i}^{*}\right)_{i=1}^{n}\right)
$$

for all $\left\{x_{i}\right\}_{i=1}^{n}$ in $X$ and all $\left\{x_{i}^{*}\right\}_{i=1}^{n}$ in $X^{*}$. This proves that the identity map $I_{X}$ is $(1, p, 1)$-summing $[\mathrm{P}]$. Hence $I_{X^{*}}$ is $(1,1, p)$-summing $[\mathrm{P}$, Theorem 17.1.5]. Since $p>2$, from [Pi2] it follows that $X^{*}$ is finite-dimensional, a contradiction which completes the proof. 
In [Ps1, Theorem 3.2], it is proved that any Banach space $E$ of cotype 2 can be imbedded isometrically into a Banach space $X$ such that $X$ and $X^{*}$ are both of cotype 2 and both verify Grothendieck's Theorem. So, if $X$ is the Banach space associated to $E=\ell_{2}$ in [Ps1, Theorem 3.2], for every $p>2$ there are $p$-summable sequences in $X$ that do not lie inside the range of a $X$-valued vector measure. Nevertheless, the closed unit ball of $\ell_{2}$ is the range of a vector measure [AD].

c) $s\left(\ell_{1}\right)=\emptyset$.

In $[\mathrm{AD}]$ it is proved that the sequence $\left(e_{n} / \sqrt{n}\right)$ does not lie inside the range of a measure. Nevertheless, $\left(e_{n} / \sqrt{n}\right)$ belongs to $\ell_{u}^{s}\left(\ell_{1}\right)$ for any $s>2$.

d) $s\left(\ell_{p}\right)=\emptyset$ for any $1<p<2$.

Given $s \in(2, q)$, we choose $\alpha \in(1 / s-1 / q, 1 / 2-1 / q)$. We are going to prove that the sequence $\left(e_{n} / n^{\alpha}\right)$ belongs to $\ell_{u}^{s}\left(\ell_{p}\right)$ but it isn't in the range of a measure. If $x^{*}=\left(x_{n}^{*}\right) \in \ell_{q}$ with $\left\|x^{*}\right\|_{q} \leq 1$, we have for all $m \in \mathbb{N}$ :

$$
\sum_{n=m}^{\infty}\left|\left\langle\frac{e_{n}}{n^{\alpha}}, x^{*}\right\rangle\right|^{s}=\sum_{n=m}^{\infty} \frac{1}{n^{\alpha s}}\left|x_{n}^{*}\right|^{s} \leq\left(\sum_{n=m}^{\infty} \frac{1}{n^{\frac{\alpha s q}{q-s}}}\right)^{\frac{q-s}{q}}\left(\sum_{n=m}^{\infty}\left|x_{n}^{*}\right|^{q}\right)^{\frac{s}{q}}
$$

where we have applied Holder's inequality. Then

$$
\sup \left\{\sum_{n=m}^{\infty}\left|\left\langle\frac{e_{n}}{n^{\alpha}}, x^{*}\right\rangle\right|^{s}:\left\|x^{*}\right\|_{q} \leq 1\right\} \leq\left(\sum_{n=m}^{\infty} \frac{1}{n^{\frac{\alpha s q}{q-s}}}\right)^{\frac{q-s}{q}}
$$

for all $m \in \mathbb{N}$. Since $\alpha s q /(q-s)>1$, it follows that $\left(e_{n} / n^{\alpha}\right)$ is plainly in $\ell_{u}^{s}\left(\ell_{p}\right)$. The sequence $\left(e_{n} / n^{\alpha}\right)$ is an unconditional basis of $\ell_{p}$; therefore, to prove that the sequence $\left(e_{n} / n^{\alpha}\right)$ is not in the range of a measure it suffices to show that it does not belong to $\ell_{w}^{2}\left(\ell_{p}\right)$ [AD, Theorem 5]. For this, choose $\beta>(1 / q)$ and consider the scalar sequence $x^{*}=\left(1 / n^{\beta}\right)$. It is plain that $x^{*} \in \ell_{q}$ and that viewed as a member of $\left(\ell_{p}\right)^{*}, x^{*}$ satisfies:

$$
\sum_{n}\left|\left\langle\frac{e_{n}}{n^{\alpha}}, x^{*}\right\rangle\right|^{2}=\sum_{n=1}^{\infty} \frac{1}{n^{2(\alpha+\beta)}} .
$$

Therefore, if we choose $\beta \in(1 / q, 1 / 2-\alpha)$, the last series is divergent and we deduce that $s \notin s\left(\ell_{p}\right)$.

e) If $X$ is an infinite-dimensional $\mathcal{L}_{p}$-space for $1 \leq p<2$, then $s(X)=\emptyset$.

By [LP, Proposition 7.3], $X$ has a complemented subspace $H$ isomorphic to $\ell_{p}$. Then $s(X) \subset s(H)=s\left(\ell_{p}\right)=\emptyset$.

In view of these results and $[\mathrm{PR}]$ a question arises: Are there Banach spaces $X$ such that $s(X) \neq \emptyset$ but $X^{*}$ is not isomorphic to a subspace of an $L_{1}(\mu)$-space for some measure $\mu$ ?

\section{REFERENCES}

[AD] R. Anantharaman and J. Diestel, Sequences in the range of a vector measure, Annales Societatis Mathematicae Polonae, Serie I: Comm. Math. XXX (1991), 221-235. MR 92g:46049

[DU] J. Diestel and J.J. Uhl, Jr, Vector measures, Math. Surveys 15, Amer. Math. Soc, Providence, R.I, 1977. MR 56:12216

[KK] I. Kluvanek and G. Knowles, Vector measures and control systems, Math. Stud., vol.20, North-Holland, 1976. MR 58:17033

[LP] J. Lindenstrauss and A. Pelczynski, Absolutely summing operators in $\mathcal{L}_{p}$-spaces and their applications, Studia Math. 29 (1968), 275-326. MR 37:6743

[LR] J. Lindenstrauss and H.P. Rosenthal, The $\mathcal{L}_{p}$ spaces, Israel J. Math. 7 (1969), 325-349.

[P] A. Pietsch, Operator Ideals, North-Holland, 1980. MR 81j:47001 
[Pi1] C. Piñeiro, Operators on Banach spaces taking compact sets inside ranges of vector measures, Proc. Amer. Math. Soc. 116 (1992), 1031-1040. MR 93b:47076

[Pi2] C. Piñeiro, Banach spaces in which every $p$-weakly summable sequence lies inside the range of a vector measure, Proc. Amer. Math. Soc., to appear. MR 96i:46016

[PR] C. Piñeiro and L. Rodriguez-Piazza, Banach spaces in which every compact lies inside the range of a measure, Proc. Amer. Math. Soc. 114 (1992), 505-517. MR 92e:46038

[Ps1] G. Pisier, Counterexamples to a conjecture of Grothendieck, Acta Mathematica 151 (1983), 181-208. MR 85m:46017

[Ps2] G. Pisier, Factorization of linear operators and geometry of Banach spaces, CBMS, vol.60, Amer. Math. Soc., Providence, R.I., 1984.

[T] N. Tomczak-Jaegermann, Banach-Mazur Distances and Finite-Dimensional Operator Ideals, vol. 38, Pitman Monographs and Surveys in Pure and Appl. Math., 1989. MR 90k:46039

Departamento de Matemáticas, Escuela Politécnica Superior, Universidad de Huelva, 21810 La RÁBida, Huelva, Spain

E-mail address: candido@colon.uhu.es 